\title{
Identification of L-asparaginases from Streptomyces strains with competitive activity and immunogenic profiles: a bioinformatic approach
}

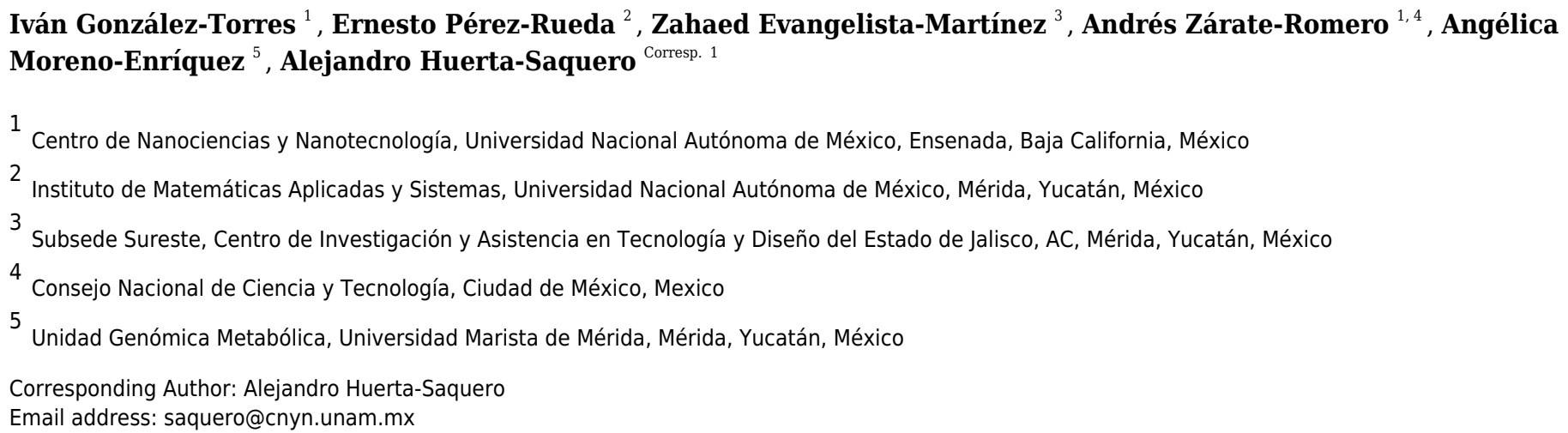

The enzyme L-asparaginase from Escherichia coli is a therapeutic enzyme that has been a cornerstone in the clinical treatment of acute lymphoblastic leukemia for the last decades. However, treatment effectiveness is limited by the highly immunogenic nature of the protein and its cross-reactivity towards L-glutamine. In this work, a bioinformatic approach was used to identify, select and computationally characterize L-asparaginases from Streptomyces through sequence-based screening analyses, immunoinformatics, homology modeling, and molecular docking studies. Based on its predicted low immunogenicity and excellent enzymatic activity, we selected a previously uncharacterized L-asparaginase from Streptomyces scabrisporus. Furthermore, two putative asparaginase binding sites were identified and a 3D model is proposed. These promising features allow us to propose L-asparaginase from $S$. scabrisporus as an alternative for the treatment of acute lymphocytic leukemia. 


\section{Identification of L-asparaginases from Streptomyces}

2 strains with competitive activity and immunogenic

3 profiles: a bioinformatic approach

4

5 Iván González-Torres ${ }^{1}$, Ernesto Perez-Rueda ${ }^{2}$, Zahaed Evangelista-Martínez ${ }^{3}$, Andrés Zárate-

6 Romero $^{1,4}$, Angélica Moreno-Enríquez ${ }^{5}$, Alejandro Huerta-Saquero ${ }^{1 *}$

7

$8{ }^{1}$ Centro de Nanociencias y Nanotecnología, Universidad Nacional Autónoma de México. Km.

9107 Carretera Tijuana-Ensenada, Ensenada, Baja California, México.

$10{ }^{2}$ Instituto de Matemáticas Aplicadas y Sistemas, Universidad Nacional Autónoma de México,

11 Mérida Yucatán, México.

$12{ }^{3}$ Centro de Investigación y Asistencia en Tecnología y Diseño del Estado de Jalisco, AC.

13 Subsede Sureste, Mérida Yucatán, México.

$14{ }^{4}$ Consejo Nacional de Ciencia y Tecnología, Ciudad de México, México.

$15{ }^{5}$ Universidad Marista de Mérida, A. C. Unidad Genómica Metabólica. Mérida, Yucatán, 16 México.

$19 *$ Corresponding author:

20 Alejandro Huerta-Saquero ${ }^{1}$

21 Km. 107 Carretera Tijuana-Ensenada, Ensenada, Baja California, México.

22 Email address: saquero@cnyn.unam.mx 


\section{Abstract}

24 The enzyme L-asparaginase from Escherichia coli is a therapeutic enzyme that has been a 25 cornerstone in the clinical treatment of acute lymphoblastic leukemia for the last decades. 26 However, treatment effectiveness is limited by the highly immunogenic nature of the protein and 27 its cross-reactivity towards L-glutamine. In this work, a bioinformatic approach was used to 28 identify, select and computationally characterize L-asparaginases from Streptomyces through 29 sequence-based screening analyses, immunoinformatics, homology modeling, and molecular 30 docking studies. Based on its predicted low immunogenicity and excellent enzymatic activity, we 31 selected a previously uncharacterized L-asparaginase from Streptomyces scabrisporus. 32 Furthermore, two putative asparaginase binding sites were identified and a 3D model is proposed. 33 These promising features allow us to propose L-asparaginase from $S$. scabrisporus as an 34 alternative for the treatment of acute lymphocytic leukemia.

\section{Introduction}

37 Acute lymphocytic leukemia (ALL) is a hematological disorder of the bone marrow and is characterized by abnormal proliferation of immature lymphoid line cells, blocked at an early stage of cell differentiation, that accumulate and replace healthy hematopoietic cells in the bone marrow (Pui, Relling \& Downing, 2004; Onciu, 2009). ALL occurs predominantly in children of 1-4 years

41 of age and represents approximately $25 \%$ of childhood cancers and about $80 \%$ of leukemias (Katz 42 et al., 2015).

43 Although in most cases the risk factors and pathogenicity associated with ALL have not been 44 clearly identified, the etiology of the disease has been mainly associated with a variety of 45 conditions; cytogenetic alterations, mutations to key genes that regulate cellular proliferation, 
46 differentiation and death; presence of oncogenic viruses, immunodeficiency, exposure to 47 pesticides, solvents, and ionizing radiation (Pui, Relling \& Downing, 2004; Bassan, Maino \& 48 Cortelazzo, 2016).

49 Treatment for ALL patients involve steroid drugs, prednisone, vincristine, and the enzyme L50 asparaginase (ASNase) (Avramis, 2012; Schwab \& Harrison, 2018). ASNase has been essential in 51 the treatment of ALL since the 1970s, with demonstrated effectiveness as an individual drug with 52 remission rates of up to $68 \%$ (Salzer et al., 2017). The combination of ASNase with other 53 anticancer drugs has led to remission rates of up to $90 \%$ (Lanvers-Kaminsky, 2017).

54 Currently, there are four ASNase formulations available for the ALL treatment: two native forms 55 of the enzyme, obtained from Escherichia coli (EcAII) and Erwinia chrysanthemi (ErAII), and 56 pegylated E. coli ASNase (EcAII-PEG), as well as pegylated E. chrysanthemi ASNase (ErAII57 PEG). Of these, EcAII-PEG has become the first-line treatments for ALL in the US, with EcAII 58 the most widely used formulation. ErAII is administered to patients who have developed 59 hypersensitivity to the above formulations (Pieters et al., 2011; Abribat, 2016; Barba et al., 2017).

60 In recent years, evidence has been accumulating of its usefulness as an important component in 61 the treatment of other hematological malignancies, such as acute myeloid leukemia, 62 myelosarcoma, lymphosarcoma, Hodgkin's disease, and chronic lymphocytic leukemia (Emadi, 63 Zokaee \& Sausville, 2014; Lopes et al., 2015). Despite their high antileukemic potential, the use 64 of ASNases in the treatment of ALL is limited by their toxicity. Among the adverse effects that 65 have been reported are leukopenia, immune suppression, acute pancreatitis, liver dysfunction, 66 hyperglycemia, abnormalities in hemostasis, and hemorrhages of the central nervous system 67 (Schein et al., 1969; Ramya et al., 2012; Chan et al., 2014; Ali et al., 2016; Hijiya \& van der Sluis, 68 2016; Kamal et al., 2019). 
69 The generation of immune responses during treatment with ASNase is a common condition that 70 has been reported in up to $75 \%$ of patients. These reactions depend on the formulation used, the 71 mode of administration (intravenous or intramuscular), and the treatment protocol (Hijiya \& van 72 der Sluis, 2015). For example, between 30 and $75 \%$ of patients that receive the native form of the

73 E. coli enzyme experience hypersensitivity reactions, and about $70 \%$ develop anti-EcAII 74 antibodies after drug administration (Battistel et al., 2020); these antibodies lead to rapid 75 inactivation of the enzyme (Walenciak et al., 2019).

76 Allergic reactions to ASNase, which are associated with its bacterial origin, range from mild 77 urticaria to life-threatening anaphylactic shock. Irritation, fever, vomiting, gastrointestinal edema, 78 and breathing difficulties are symptoms frequently reported (Lanvers-Kaminsky, 2017). On the 79 other hand, adverse effects have been reported due to the toxicity produced by 80 glutaminase cross activity, such as leukopenia, immune suppression, acute pancreatitis,

81 hyperglycemia, thrombosis, neurotoxicity, and liver failure, among others (Ramya et al., 2012;

82 Chan et al., 2014; Ali et al., 2016).

83 Different strategies to reduce the toxicity of ASNase have been reported, including modifications 84 in the structure of the protein by mutagenesis, design of mutants with diminished ability to 85 hydrolyze L-glutamine, chemical modifications in specific amino acids, and modifications to drug 86 formulations (Ramya et al., 2012; Nguyen, Su \& Lavie, 2016; Nguyen et al., 2018). Covalent 87 conjugation of the enzyme with polyethylene glycol, known as PEGylation, reduces the incidence 88 of hyperglycemia, pancreatitis, and anaphylaxis. This specific modification increase the half-life 89 of the enzyme and reduces the frequency of drug administration (Thomas \& Le Jeune, 2016).

90 On the other hand, the exploration of new sources of ASNases offers the possibility of finding 91 versions of the enzyme with different pharmacological characteristics, potentially useful for the 
92 treatment of ALL and other lymphomas (Krishnapura, Belur \& Subramanya, 2016). In this sense,

93 besides searching for less immunogenic asparaginases, it is essential to find those with high affinity

94 for L-asparagine (in the micromolar range) in order to have the potential for therapeutic use. Some 95 atypical ASNases, unrelated to EcAII and ErAII, such as Rhizobium etli type II ASNase (ReAII) 96 (Ortuño-Olea \& Durán-Vargas, 2000), have been proposed as alternatives with therapeutic 97 potential; the $R$. etli ASNase has null glutaminase activity and a different immunogenic profile 98 than E. coli and E. chrysantemy ASNases (Moreno-Enriquez et al., 2012; Huerta-Saquero et al., 99 2013). However, this enzyme has a low affinity for asparagine, which limits its use. Despite the 100 success of E. coli and E. chrysanthemi ASNases in therapeutic regimens for ALL and other types 101 of leukemia, the search for new ASNases that are less toxic and less immunogenic is necessary. In 102 this sense, ASNases from phylogenetically distant microorganisms offer a specific target for the 103 selection of variants with the appropriate characteristics. Among these, ASNases from 104 Streptomyces are one potential group to be evaluated for immunogenicity, toxicity, and affinity for 105 L-asparagin to obtain new ASNases with therapeutic potential. The main characteristics to select asparaginases with therapeutic potential are the high affinity for L-asparagine (in the micromolar range), null or low glutaminase cross-activity, as well as a different three-dimensional folding from the E. coli asparaginase, which suggests different immunogenicity. In this work, we develop a strategy based on bioinformatics tools to analyze and select ASNases from Streptomyces for ALL treatment, taking advantage of its phylogenetic distance from E. coli, looking for those candidates

111 that meet the two fundamental criteria: asparaginases with high affinity for asparagine (using active site prediction tools and molecular docking), and that have lower immunogenicity (using 113 antigenicity and protein structure prediction tools). As a reference, we selected the E. coli and 114 Streptomyces coelicolor ASNases. The importance of this novel approach is discussed. 


\section{Materials \& Methods}

\section{Identification and selection of homologous L-Asparaginases}

118 Putative ASNases from Streptomyces were identified through a BLASTp search against the NR 119 database of the NCBI using as seeds the amino acid sequences of EcAII (ID P00805) and Streptomyces coelicolor type II ASNase (ScAII; ID Q9K4F5). The search was restricted to the Streptomyces taxon (Taxid 1883), and an E-value less than 1e-06 was considered significant.

Partial proteins and those from unidentified Streptomyces strains were excluded. In a posterior step, the set of protein sequences was filtered at $60 \%$ identity as cutoff to avoid redundancy, using the CD-Hit program (http://weizhongli-lab.org/cdhit_suite/cgi-bin/index.cgi) (Huang et al., 2010).

Each cluster was analyzed using the HMMER program on the PFAM server

126 (http://pfam.xfam.org/) to determine the protein family to which they belonged (Finn, Clements \& 127 Eddy, 2011; Finn et al., 2016).

\subsection{Phylogenetic analysis}

130 ASNases amino acid sequence alignments were performed using Clustal Omega (Sievers et al., 131 2011) with default parameters. The quality of the alignments was improved using the model PF06089.11 or PF00710.11 of ASNase, as required. Multiple sequence alignment statistics were computed with AliStat (http://www.csb.yale.edu/userguides/seq/hmmer/docs/node27.html). 
138 EcA but it does not have therapeutic potential. For the PF06089.11 cluster, Rhizobium etli type II

139 ASNase (ReAII) was included in the analysis.

$140 \quad 1.3$ Antigenicity prediction

141 The prediction of the probability of antigenicity of each ASNase was calculated with the server

142 ANTIGENpro (http://scratch.proteomics.ics.uci.edu/) (Magnan et al., 2010). ANTIGENpro is a

143 sequence-based, alignment-free, protein antigenicity predictor with an estimated accuracy of $82 \%$.

\subsection{HLA class II binding prediction}

146 The amino acid sequence of each candidate ASNase was screened for T-cells epitopes with the 147 MHC II Analysis Resource at the Immune Epitope Data Base (IEDB) server 148 (http://tools.iedb.org/mhcii/). MHC II Analysis Resource parses sequences into 15-mer and 149 assesses the binding potential of each 15-mer to MHC class II molecules of one or more HLA 150 alleles. The IEDB recommended method was used for predictions for a set of 8 HLA alleles that 151 collectively represent >\%95 world population: HLA-DRB1*01:01, HLA-DRB1*03:01, HLADRB1*04:01, HLA-DRB1*07:01, HLA-DRB1*08:01, HLA-DRB1*11:01, HLA-DRB1*13:01

153 and HLA-DRB1*15:01. The IEDB-recommended method uses the consensus approach, 154 combining NN-align, SMM-align, CombLib, Sturniolo, and NetMHCIIpan (Wang et al., 2010).

155 For each peptide, a percentile rank is generated by comparing the peptide's score against the scores 156 of five million random 15-mer selected from SWISSPROT database, and the median percentile 157 rank is used to calculate a consensus percentile rank (CPR). Peptides with a CPR $<2$ were defined 158 as high-affinity binders and thus selected for epitope density (ED) calculation. Multiple 9-mer 159 cores were identified in overlapped 15-mer peptides. To reduce overestimation of predicted 160 peptides, only the 9-mer cores, predicted by using the Sturniolo method (Sturniolo et al., 1999) 
161 and with a CPR $<1$, were considered for the analysis. Finally, epitope density (ED) was calculated

162 using the follow equation, modified from (Santos et al., 2013):

163

164

$$
E D=\frac{\text { Predicted epitope } *(2-\text { Affinity average }(\mathrm{cpr}))}{\text { Protein length size }- \text { Epitope size }+1}
$$

Where Predicted epitope is the number of epitopes with a CPR $<1$.

166

167

Epitope coverage was calculated as the number of alleles covered by the epitope consensus, according to the following assumption: when a small number of alleles is covered, a lower percentage of the population will develop sensitivity to ASNase.

\subsection{Protein structure prediction, refinement and quality assessment}

172 The three-dimensional structures of the selected ASNases was modeled by homology using the I-

173 Tasser server (https://zhanglab.ccmb.med.umich.edu/I-TASSER/) (Zhang, 2008). In brief,

174 starting from an amino acid sequence, I-Tasser generates three-dimensional atomic models from

175 multiple threading alignments and iterative structural assembly simulations. A C-score, provided

176 as an estimate of the accuracy of the models generated, typically ranges between -5 to +2 , with a

177 higher value indicating higher confidence, and vice versa (Roy, Kucukural \& Zhang, 2010).

178 For each ASNase, the model with the higher C-score was selected and then refined using the

179 ModRefiner server (https://zhanglab.ccmb.med.umich.edu/ModRefiner/). ModRefiner improves

180 the physical quality and structural accuracy of three-dimensional protein structures by a two-step, 181 atomic-level energy minimization (Xu \& Zhang, 2011). Finally, the quality of the models was 182 evaluated by RAMPAGE (http://mordred.bioc.cam.ac.uk/ rapper/rampage.php), Qmean 183 (https://swissmodel.expasy.org/qmean/), and Verify3D (http:/servicesn.mbi.ucla.edu/Verify3D). 
185

186

187

188

189

190

191

192

193

194

195

196

197

198

199

200

201

202

203

204

\subsection{Molecular docking}

The molecular coupling was carried out using Autodock Tools software (Sanner, 1999; Morris et al., 2009). EcAII (PDB ID: 3ECA) was recovered from the PDB protein database (http://www.rcsb.org/) (Swain et al., 1993; Berman et al., 2000). Once refined, selected ASNase structures were prepared using Dock prep at UCSF Chimera and refined using the Gasteiger method (Gasteiger and Marsili, 1978).

The three-dimensional structures of the asparagine and glutamine ligands were obtained from the DrugBank repository (https://www.drugbank.ca/; accession numbers DB00174 and DB00130, respectively) (Wishart et al., 2018). The preparation of the ligands was carried out by the Gasteiger method and, finally, the allocation of the rotation centers was determined (Gasteiger and Marsili, 1978).

For each ASNase, the search box was focused on previously proposed active sites. The box size was defined to cover all residues of the ligand binding site, using a grid size of $0.375 \AA$.

Blind molecular docking was performed with Autodock 4.2 software, using the Lamarckian genetic algorithm, with 1000 runs, for a population size equal to 150 , with $2.5 \times 10^{\wedge} 6$ evaluations, a mutation rate equal to 0.02 in 27,000 generations.

In addition, the active site location was predicted by AutoLigand (Harris, Olson \& Goodsell, 2008). Briefly, AutoLigand identifies sites of maximum affinity from maps generated by AutoGrid, finding regions with better energy and a lower volume. 


\section{Results}

206 2.1 L-Asparaginases from Streptomyces cluster into two type families according to its protein 207 architecture

208 The Blast search against the Streptomyces taxon revealed 296 putative ASNases homologous to 209 EcAII and 703 homologous to ScAII with a significant score. After manual examination of both 210 groups, 136 and 311 complete sequences were kept for EcAII and ScAII groups, respectively. 211 Protein domain analysis using PFAM server showed that 136 sequences are related to the 212 PF00710.11 family of N-terminal ASNases. For sequences homologous to ScAII, PFAM analysis 213 revealed that they belong to the PF06089.11 family of ASNases, a group of enzymes related to 214 ReAII, a thermolabile enzyme induced by L-asparagine and repressed by the carbon source 215 (Moreno-Enriquez et al., 2012; Huerta-Saquero et al., 2013). Representative clusters for 216 PF00710.11 and PF06089.11 families obtained using the CD-Hit suite program were generated at 217 a 60\% identity cutoff, with 19 and 7 putative ASNases, respectively (Table 1). ASNases sequences 218 showed similar lengths in both clusters, ranging from 320 to 420 amino acids.

219 The sequences belonging to the PF00710.11 family have conserved residues located at the ligand 220 binding site necessary for L-asparagine hydrolysis: Thr 12, Tyr 25, Ser 58, Gln 59, Thr 89, Asp 221 90, and Lys 162 for subunit A; Asn 248 and Glu 283 for subunit C. In this regard, Thr 12-Lys 222 162-Asp 90 and Thr 12-Tyr 2-Glu 283 are the catalytic triads involved in L-asparagine 223 hydrolysis, where Thr 12 and Thr 89 are involved in the nucleophilic attack of the substrate (Gesto 224 et al., 2013; Sanches, Kraunchenko \& Polikarpov, 2016).

225 Concerning the PF06089.11 family, we identified an N-terminal conserved motif, with sequences 226 NCSGKHxAM, DGCGAPL, SHSGEx(2)H, and PRSx(2)KPxQ probably involved in asparagine 227 hydrolysis. ReAII hydrolyzes L-asparagine at similar levels to Erwinia chrysanthemi, but with 
228 lower affinity than L-asparaginases from both E. coli and E. chrysanthemi (Moreno-Enriquez et 229 al., 2012). Furthermore, ReAII is the only ASNase characterized from the PF06089.11 family

230

$231 \quad 2.2$ Phylogenetic analysis of ASNases

232 For the PF00710.11 family, EcAI was added to the multiple sequence alignment in order to know 233 the relationship between this ASNase and the candidate ASNases. EcAI belongs to the same family 234 of proteins as EcAII, but it does not represent a therapeutic option for ALL treatment. It is 235 noteworthy that asparaginases can also be classified according to subcellular localization, a) 236 periplasmic asparaginases containing secretion signal peptide and, b) asparaginases with 237 intracellular localization. The former generally have a higher affinity for asparagine. However, 238 according to their architecture, both types of proteins can be found in the PF00710.11 or 239 PF06089.11 families. This is the case of E. coli asparaginases I and II, both belonging to the 240 PF00710.11 family (https://pfam.xfam.org/family/PF00710\#tabview=tab1). We found that the 241 ASNase with accession number WP_059134811.1 of Streptomyces alboniger is grouped in the 242 same clade as EcAI, and so it was excluded from subsequent analyses (Figure 1A).

243 The phylogenetic reconstruction showed three well-defined clades (Figure 1A). The first clade 244 includes ASNases from Streptomyces species S. aureocirculatus (WP_078965752.1), S. cattleya 245 (WP_014151616.1), S. thermoautotrophicus (KWW98572.1), S. himastatinicu (EFL23513.1), S. 246 turgidiscabies (ELP65653.1), S. nanshensis (WP_070201703.1), and S. griseus 247 (WP_030748190.1).

248 The second clade includes ASNases from S. albidoflavus (WP_095730579.1), S. kebangsaanensis 249 (WP_073950513.1), S. fradiae (WP_078649241.1), S. himastatinicus (WP_009718687.1), S. 250 purpureus (WP_078513220.1), and S. paucisporeus (WP_079189481.1). Finally, the third clade 
251 contains proteins from S.purpurogeneiscleroticus (WP_053609500.1), S. purpurogeneiscleroticus

252 (WP_053610569.1), S. phaeochromogenes (WP_055617501.1), and S. lavenduligriseus

253 (WP_051815467.1) where EcAII was included, suggesting that proteins clustered in this clade

254 share similar properties to EcAII. In addition, two proteins, WP_053609500.1 and

255 WP_055617501.1, exhibited the largest proportion of antigenic regions, with almost the same

256 probability regions as the EcAII protein.

257 On the other hand, for the ASNases of PF06089.11, phylogenetic analysis included both the 258 ASNase sequence of $R$. etli and S. coelicolor (ReAII and ScAII, respectively) (Figure 1B). The 259 tree defines two clades. In the first one, where the ScAII was included, we also considered 260 ARZ68596.1 from S. albireticuli, SOD64826.1 from S. zhaozhouensis, WP_078645645 from 261 S. varsoviensis, CDR15801.1 from S. iranensis, and WP_020554088 from S. scabrisporus. 262 In the second clade were included the following proteins: WP_044373749 from S. ahygroscopicus 263 and WP_078980718.1 from S. scabrisporus.

264

265 Figure 1. Phylogenetic tree of PF00710.11 (A) and PF06089.11 (B) families. Blue dots highlight 266 reference sequences added to each analysis. Red dots highlight sequences used as internal controls 267 (asparaginases from E. coli and R. etli, respectively). A total of 1000 replicates were performed. 268 Bootstrap values are indicated.

269

\section{$270 \quad 2.3$ Antigenicity predictions}

271 The results for antigenicity showed a likelihood of being antigenic for all ASNases in both sets

272 that was lower than that of EcAII (Figure 2). Nevertheless, among selected Streptomyces ASNases,

273 the candidates from S. purpurogeneiscleroticus (WP_053609500.1) and S. phaeochromogenes 
274 (WP_055617501.1) showed a higher probability of being antigenic, whereas the rest of the

275 ASNases showed very low antigenicity values in comparison with an E. coli ASNase 276 (P00805_EcAII).

277

278 Figure 2. ASNase antigenicity predictions. The antigenicity scores for PF00710.11 family (A) and 279 PF06089.11 family (B) of Streptomyces asparaginases were compared with the EcAII antigenicity 280 score.

281

\section{$282 \quad 2.4$ T-cell epitope analysis}

283 After antigenicity prediction, the ED, the total number of high-affinity epitopes, the affinity 284 epitopes, and the number of HLA alleles covered by each ASNase were calculated. The results 285 showed that the ASNases with accession numbers WP_053609500.1, WP_053610569.1, 286 EFL23513.1, WP_095730579.1, WP_078513220.1, and WP_052425051.1 have higher EDs than 287 the reference (P00805_EcAII; ED=0.01114; 5 covered alleles) (Figure 3).

288 On the other hand, the ASNase with the lowest predicted ED was WP_044373749.1, with an ED 289 of 0.0027 and a coverage of 4 alleles, following by WP_095730579.1 (2 alleles), ELP65653.1 (3 290 alleles), and Q9K4F5 (3 alleles) (Table 2).

291 Additionally, the distribution of epitopes was mapped into the sequences of the ASNases (Figure 292 3). ASNases of the PF06089.11 family tended to have a lower ED (Table 3) as well as lower allele 293 coverage than those of the PF00710.11 family (Figure 3). 
295 Figure 3. Epitope mapping of ASNases of the PF familes evaluated, PF00710.11 and PF06089.11.

296 The epitopes identified along with the ASNase sequences are shown. The color intensity represents 297 the number of hits for each of them.

298

299 Next, ASNases with lower allele coverage, lower ED, and lower probability of antigenicity were 300 selected for further analysis. S. coelicolor (Q9K4F5), S. scabrisporus (WP_078980718.1), and S. 301 albireticuli (ARZ68596.1) were selected as promising enzymes.

302

303

\subsection{Protein structure predictions}

304 From selected ASNases, homology-based models were generated (I-Tasser). For the subsequent 305 analysis, the S. scabrisporus asparaginase II model, which had the highest C-value, was chosen 306 (WP_078980718.1 SsAII-2) (Figure 4). The structural model obtained by I-Tasser (with a C-value 307 of -3.09) was refined with ModRefiner. In addition, the RAMPAGE program 308 (http://mordred.bioc.cam.ac.uk/ rapper) and Verify3D were used to validate the stereochemical 309 quality of the resulting three-dimensional model. After analyzing the Ramachandran plot, 91.7\%

310 and $5.5 \%$ of the residues were located in favored and allowed regions, respectively; whereas

311 Verify3D analysis revealed that $80.73 \%$ of the residues had an average 3D-1D score $<0.2$, 312 indicating that the model is compatible with its sequence.

313 Based on the predicted structure, ASNase WP_0789718.1 (PF06089.11 family) is related in terms 314 of folding to the beta-lactamase family. Beta-lactamases (SCOP data base, entry 56600) consist of 315 a cluster of alpha-helices and an alpha/beta sandwich. This folding is also found in transpeptidases, 316 esterases, penicillin receptors, D-aminopeptidases, and glutaminases (InterPro IPR012338). 
318 Figure 4. 3D protein structure prediction of S. scabrisporus asparaginase II (WP_078980718.1;

319 SsAII-2).

320

\section{$321 \quad 2.6$ Active site prediction}

322 In order to identify the active site residues of the S. scabrisporus ASNase (WP_0789718.1), three

323 approaches were used: genomic comparison, blind molecular coupling simulation, and search for 324 high-affinity binding pockets with AutoLigand (active site). To our knowledge, there is no 325 information regarding the active site of the family PF06089.11 ASNases, so genomic comparison 326 was not possible. Using AutoLigand, two possible high affinity binding sites for L-asparagine were 327 identified (Figure 5A). The first (site A) had a volume of $121 \AA^{3}$ and an energy per volume equal 328 to $-0.2149 \mathrm{kcal} / \mathrm{mol} \AA^{3}$; the second (site B) had a volume of $101 \AA^{3}$ and an energy per volume 329 equal to $-0.2136 \mathrm{kcal} / \mathrm{mol} \AA^{3}$. Site $\mathrm{A}$ is located between an alpha-helix in the amino terminal 330 containing the ${ }^{57} \mathrm{PRSx}(2) \mathrm{KPxQ}^{65}$ motif, and a loop in the central region of the enzyme, containing 331 the ${ }^{141}$ NCSGKHxAML ${ }^{150}$ motif (Table 3 ). Site B is located in a pocket formed by a set of alpha332 helices in the amino terminal of the protein, marked by the presence of the ${ }^{87}$ SHTGQxHFV ${ }^{95}$ motif. 333 On the other hand, by performing AutoDock 4.2 whole-protein molecular coupling simulations, 334 we found that the best ligand-enzyme interaction (L-asparagine-ASNase), with a binding free 335 energy of $-4.17 \mathrm{kcal} / \mathrm{mol}$, targeted residues corresponding to the ${ }^{141} \mathrm{NCSGKHxAML}{ }^{150}$ motif, 336 which correspond to the site A (Figure 5B).

337

Figure 5. SsAII-2 putative binding sites. A) Site A (orange) contains the NCSGKHxAML 339 sequence and site B (blue) contains the SHTGQxHFV motif. B) Residues involved with asparagine

340 through a direct interaction, obtained by blind molecular docking. 
342 Additionally, in order to validate AutoLigand analysis searching active sites in the S. scabrisporus 343 ASNases, a search for binding sites in EcAII was performed. To do this, the monomeric, dimeric, 344 and tetrameric forms of the enzyme (the latter is the catalytically active form) were analyzed using 345 the same conditions used for SsAII-2. It was found that AutoLigand successfully identified the 346 binding site of L-Asn, consisting of Thr 12, Tyr 25, Ser 58, Gln 59, Thr 89, Asp 90, and Lys 162 347 and also Asn 248 and Glu 283 (Figure 6), the latter two only for dimeric and tetrameric forms. The 348 sites found (red squares curves) had energies by volume equal to $-0.2119,-0.2242$, and -0.2366 $349 \mathrm{kcal} / \mathrm{mol} \AA^{3}$ and a volume of 136,122 , and $102 \AA^{3}$ for the monomer, dimer, and tetramer, 350 respectively (Figure 7). It is relevant that for both the dimeric and the tetramer forms, AutoLigand 351 successfully identified L-Asn binding pockets in EcAII: the pocket formed between the amino352 terminal end of subunit $\mathrm{A}$ and the carboxy terminal of the subunit $\mathrm{C}$, as well as equivalent pockets 353 for dimer BD. In addition, several other solutions found by AutoLigand (curve with blue or green 354 squares), using up to 90 filling points, converge in the different joint pockets formed by dimers.

Figure 6. EcAII dimer AutoLigand analysis. Cyan EcAII subunit C is shown in cyan and subunit A in magenta. The red mesh represents the highest-affinity pocket found by AutoLigand (putative active site). The site represented in the scheme corresponds to the residues located at a maximum distance of $5 \AA$ using 20 points: Thr 12, Tyr 25, Ser 58, Gln 59, Thr 89, and Asp 90 from subunit C and Asn 248 and Glu 283 from subunit A.

361

362 Figure 7. AutoLigand results for EcAII. The minima observed in the total energy graphs per unit 363 volume represent putative binding sites in the structures analyzed, for the monomer, dimer, and 
364 tetramer conformation. As more filling points are used, the binding sites, cavities, or grooves are 365 filled and the affinity decreases. The best sites are the ones with the lowest energy and the lowest 366 volume.

367

368

\subsection{Molecular docking}

369 370

371

372 373

374 375 376 377 378 379 380 381 382 383 384 385
Molecular docking simulations were performed at the putative sites found (Table 4). For EcAII, as the reference ASNase, Thr 12, Tyr 25, Ser 58, Gln 59, Thr 89, Asp 90, Asn 248, and Glu 283 were established as flexible residues; meanwhile, molecular docking for S. scabrisporus ASNase were performed using only the rigid structure of the protein, without defining flexible side chains for L-asparagine binding.

Our results showed a higher affinity for L-asparagine of the S. scabrisporus ASNase site A than site B; however, the affinity was lower than that for EcAII. For S. scabrisporus ASNase site A, the L-asparagine interacts with residues Ser 59, Lys 62, Asn 141, Ser 143, Lys 145, His 146, Gly 237, Lys 255, and Gly 256 (Figure 8A); for site B, the residues that interact with L-asparagine are Ala 84, Gly 78, Ser 87, Tyr 163, Leu 164, and Asp165 (Figure 8B). Interestingly, from site A, Lys 62, Asn 141, Ser 143, Lys 145, and His 146 are highly conserved in ASNases of the PF06089.11 family.

Figure 8. Interaction maps for sites A and B from S. scabrisporus ASNase. The black spheres represent carbon atoms, the blue nitrogen and the red oxygen. Hydrogen bonds are represented by green dotted lines and hydrophobic interactions are shown as red half-moons.

\section{Discussion}


387 In this work, a set of bioinformatics tools were used to identify, select, and characterize ASNases

388 from the Streptomyces genus. ASNase identification was carried out by searching sequences

389 homologous to EcAII and ScAII. EcAII is the best-characterized and most widely used ASNase

390 for ALL treatment, while ScAII is a homologous ASNase related to ReAII, an atypical ASNase

391 with no glutaminase activity and with a different immunogenic profile than EcAII (Huerta-Saquero

392 et al., 2013). The search for homologous sequences resulted in two sets of sequences with a high

393 probability of being ASNases (E value $<1 \mathrm{e}-06$ ). These sequence sets, in turn, were classified into

394 two different protein families based on their homology, using HMMer: PF00710.11 and

395 PF06089.11, according to the classification of the PFAM database. So far, most of the reported

396 ASNases belong to the PF00710.11 family and have been extensively studied. EcAII and the $E$.

397 chrysanthemi ASNase belong to this family. On the other hand, the PF06089.11 family represents

398 a group of atypical ASNases that remain poorly characterized. Some representative reports about

399 these ASNases include the R. etli ASNase (Ortuño-Olea \& Durán-Vargas, 2000; Moreno-Enriquez

400 et al., 2012; Huerta-Saquero et al., 2013).

401 Interestingly, the BLAST results showed a greater abundance of PF06089.11 family sequences

402 compared to the PF00710.11 family in Streptomyces. In addition, we found that about $20 \%$ of

403 species have ASNase isoforms. In that sense, many Gram-negative bacteria have at least two

404 isozymes of the family PF00710.11 (Fernández \& Zúñiga, 2006) and, in E. coli, the existence of a

405 third isoenzyme has been recently reported (Correia da Silva et al., 2018). Historically, the genus

406 Streptomyces has been attractive due to the wide repertoire of bioactive molecules produced.

407 However, searching for ASNases of pharmacological interest has been done only rarely.

408 After the identification of two sets of ASNases, we chose T-cell ED as the immunogenicity

409 indicator, according to Cantor et al. (2004), Fernández et al. (2012), and Galindo-Rodríguez et al. 
410 (2017), who proposed that HLA class II molecules play a critical role in the development of

411 specific anti-ASNase antibodies and in hypersensitivity to the enzyme (Cantor et al., 2011;

412 Fernandez et al., 2014; Galindo-Rodríguez et al., 2017). Additionally, it has been shown that

413 proteins that are highly immunogenic generally contain a greater amount of T-cell epitopes, or

414 clusters thereof (Singh et al., 2012). In addition, the measurement and prediction of ED have

415 generated interest as useful tools for comparisons between therapeutic proteins, allowing selection

416 of the best candidate in terms of probable immunogenicity (De Groot \& Martin, 2009). In this

417 sense, our results showed that ASNases of the PF06089.11 family contain lower EDs than enzymes

418 of the PF00710.11 family, as well as fewer epitope clusters throughout the sequence. In addition,

419 the allele coverage, which is related to the percentage of the population that develops a significant

420 immune response, showed Streptomyces ASNases to be potential pharmacological options. In

421 other words, due to their low content of T-cell epitopes, low antigenicity profile, and low allele

422 coverage, Streptomyces ASNases represent, in terms of immunogenicity, a pharmacological

423 alternative for ALL treatment. In this sense, the Streptomyces brollosae NEAE-115 ASNase has

424 better cytotoxicity and immunogenicity profiles for use in ALL treatment, based on evaluation in

425 a murine model, compared with EcAII (El-naggar et al., 2018). Previously, anticancer activity of

426 the Streptomyces fradiae NEAE-82 ASNase in colon cancer cell cultures was reported (El-Naggar

427 et al., 2016).

428 For the PF06089.11 family of ASNases, the lack of information of the active site precludes direct

429 comparison, as was used in the approach for the ASNase WP_078979039.1. However, the use of

430 computational tools based on structure inspection and on the evaluation of affinity maps has

431 proven highly effective in identifying probable binding sites in uncharacterized proteins (Harris,

432 Olson \& Goodsell, 2008). Based on the use of computational tools, it was possible to identify two 
433 putative binding sites in SsAII-2 (WP_078980718.1). Interestingly, in both sites the motifs

434 NCSGKHxAM, PRSx(2)KPxQ, and SHTGQx(2)H were identified, and these motifs are highly 435 conserved in the PF06089.11 family (Moreno-Enriquez et al., 2012). Of these., Borek et al. (2001)

436 proposed that some of the residues of the NCSGKHxAM motif could be involved in the hydrolytic

437 deamidation of L-asparagine (Borek \& Jaskólski, 2001).

438 On the other hand, the residues we found conserved in this family of asparaginases resemble those 439 of the active site of the Ntn amidotransferases, in which, among the important residues for 440 glutamine deamidation are found Cys, Asn, and Gly, all of them present in NCSGKHxAM motif, 441 and the deamidation mechanism proceeds with an oxyanion formation with the substrate. Although 442 this mechanism is described for glutamine amidohydrolases, it may be a mechanism similar to that 443 of this family of asparaginases, whose active site is different from those of the PF00710.11 family 444 (Isupov et al., 1996).

445 Although site A showed higher affinity for L-asparagine binding, additional studies are needed to 446 confirm the best site for ligand binding. Additionally, molecular dynamics simulations can provide 447 more evidence of the characteristics of the binding site and, together with in vitro studies, will be 448 useful for understanding the mechanism of enzymatic reaction (Karplus \& Kuriyan, 2005). 449 Although our results predicted that SsAII-2 has a lower affinity than EcAII, its different folding 450 and immunogenic characteristics place it as a good candidate. Identifying catalytic site residues 451 will allow us to perform site-directed modifications to increase its affinity.

452 The strategy developed here can be applied to the search for asparaginases in other clades of 453 microorganisms, and even in eukaryotes, specifically mammalian asparaginases, whose 454 evolutionary proximity to humans predicts less immunogenicity. 


\section{Conclusions}

457 In summary, the search for ASNases in phylogenetically distant microorganisms and the 458 application of bioinformatic tools to assess their toxicity and affinity for L-asparagine are viable 459 approaches to obtain new ASNases with therapeutic potential. Based on its low immunogenicity 460 and excellent enzymatic activity predicted, we have identified the $S$. scabrisporus ASNase as a 461 potential alternative for the treatment of ALL. The subsequent enzymatic and immunogenic 462 characterization of the S. scabrisporus ASNase is necessary for the validation of this bioinformatic 463 approach.

464

\section{Acknowledgements}

466

We acknowledge Katrin Quester and Itandehui Betanzo for technical assistance.

468

469

470

\section{References}

472

473 Abribat T. 2016. Pegylated L-asparaginase. 1:1-5.

474 Ali U, Naveed M, Ullah A, Ali K, Shah SA, Fahad S, Mumtaz AS. 2016. L-asparaginase as a 475 critical component to combat Acute Lymphoblastic Leukaemia (ALL): A novel approach to 476 target ALL. European Journal of Pharmacology 771:199-210. DOI:

$477 \quad$ 10.1016/j.ejphar.2015.12.023.

478 Avramis VI. 2012. Asparaginases: biochemical pharmacology and modes of drug resistance. 
479

480

481

482

483

484

485

486

487

488

489

490

491

492

493

494

495

496

497

498

499

500

501

Anticancer research 32:2423-37.

Barba P, Dapena JL, Montesinos P, Rives S. 2017. Asparaginasas en el tratamiento de la leucemia linfoblástica aguda. Medicina Clínica 148:225-231. DOI: 10.1016/j.medcli.2016.12.006.

Bassan R, Maino E, Cortelazzo S. 2016. Lymphoblastic lymphoma: an updated review on biology, diagnosis, and treatment. European Journal of Haematology 96:447-460. DOI: 10.1111/ejh.12722.

Battistel AP, Rocha BS da, Santos MT dos, Daudt LE, Michalowski MB. 2020. Allergic reactions to asparaginase: Retrospective cohort study in pediatric patients with acute lymphoid leukemia. Hematology, Transfusion and Cell Therapy. DOI: 10.1016/j.htct.2019.10.007.

Berman HM, Westbrook J, Feng Z, Gilliland G, Bhat TN, Weissig H, Shindyalov IN, Bourne PE. 2000. The Protein Data Bank. Nucleic Acids Research 28:235-242. DOI: $10.1093 /$ nar/28.1.235.

Borek D, Jaskólski M. 2001. Sequence analysis of enzymes with asparaginase activity. Acta Biochimica Polonica 48:893-902.

Cantor JR, Yoo TH, Dixit A, Iverson BL, Forsthuber TG, Georgiou G. 2011. Therapeutic enzyme deimmunization by combinatorial T-cell epitope removal using neutral drift. Proceedings of the National Academy of Sciences 108:1272-1277. DOI: 10.1073/pnas.1014739108.

Chan WK, Lorenzi PL, Anishkin A, Purwaha P, Rogers DM, Sukharev S, Rempe SB, Weinstein JN. 2014. The glutaminase activity of L- Asparaginase is not required for anticancer activity against ASNS-negative cells. Blood 123:3596-3606. DOI: 10.1182/blood-2013-10-535112. 
502 Correia da Silva R, Santos Siqueira A, Ranieri Jerônimo Lima A, de Melo Lima A, Silva Santos

503 A, Cristina Figueira Aguiar D, Costa Gonçalves E. 2018. In silico characterization of a

504 cyanobacterial plant-type isoaspartyl aminopeptidase/asparaginase. DOI: 10.1007/s00894$505 \quad 018-3635-6$.

506 El-naggar NE, Deraz SF, El-ewasy SM, Suddek GM. 2018. Purification, characterization and 507 immunogenicity assessment of glutaminase free L-asparaginase from Streptomyces 508 brollosae NEAE-115. :1-15.

509 El-Naggar NE-A, Deraz SF, Soliman HM, El-Deeb NM, El-Ewasy SM. 2016. Purification, 510 characterization, cytotoxicity and anticancer activities of L-asparaginase, anti-colon cancer 511 protein, from the newly isolated alkaliphilic Streptomyces fradiae NEAE-82. Scientific $512 \quad$ Reports 6:32926. DOI: 10.1038/srep32926.

513 Emadi A, Zokaee H, Sausville EA. 2014. Asparaginase in the treatment of non-ALL hematologic 514 malignancies. Cancer Chemotherapy and Pharmacology 73:875-883. DOI:

$515 \quad 10.1007 / \mathrm{s} 00280-014-2402-3$.

516 Fernandez CA, Stewart E, Panetta JC, Wilkinson MR, Morrison AR, Finkelman FD, Sandlund 517 JT, Pui CH, Jeha S, Relling M V., Campbell PK. 2014. Successful challenges using native 518 E. coli asparaginase after hypersensitivity reactions to PEGylated E. coli asparaginase. 519 Cancer Chemotherapy and Pharmacology 73:1307-1313. DOI: 10.1007/s00280-014-2464$520 \quad 2$.

521 Fernández M, Zúñiga M. 2006. Amino acid catabolic pathways of lactic acid bacteria. Critical 522 Reviews in Microbiology 32:155-183. DOI: 10.1080/10408410600880643.

523 Finn RD, Clements J, Eddy SR. 2011. HMMER web server: interactive sequence similarity $524 \quad$ searching. Nucleic Acids Research 39:W29-W37. DOI: 10.1093/nar/gkr367. 
525 Finn RD, Coggill P, Eberhardt RY, Eddy SR, Mistry J, Mitchell AL, Potter SC, Punta M,

526 Qureshi M, Sangrador-Vegas A, Salazar GA, Tate J, Bateman A. 2016. The Pfam protein

527 families database: towards a more sustainable future. Nucleic Acids Research 44:D279_

528 D285. DOI: 10.1093/nar/gkv1344.

529 Galindo-Rodríguez G, Jaime-Pérez JC, Salinas-Carmona MC, González-Díaz SN, Castro-Corona

530 Á, Cavazos-González R, Treviño-Villarreal H, Heredia-Salazar AC, Gómez-Almaguer D.

531 2017. Do immunoglobulin G and immunoglobulin E anti- 1 -asparaginase antibodies have

532 distinct implications in children with acute lymphoblastic leukemia? A cross-sectional

533 study. Revista Brasileira de Hematologia e Hemoterapia 39:202-209. DOI:

$534 \quad$ 10.1016/j.bjhh.2016.11.006.

535 Gasteiger J, Marsili M. 1978. A new model for calculating atomic charges in molecules.

536 Tetrahedron Letters 19:3181-3184. DOI: 10.1016/S0040-4039(01)94977-9.

537 Gesto DS, Cerqueira NMFSA, Fernandes PA, Ramos MJ. 2013. Unraveling the enigmatic

538 mechanism of L-asparaginase II with QM/QM calculations. Journal of the American

$539 \quad$ Chemical Society 135:7146-58. DOI: 10.1021/ja310165u.

540 De Groot AS, Martin W. 2009. Reducing risk, improving outcomes: Bioengineering less

541 immunogenic protein therapeutics. Clinical Immunology 131:189-201. DOI:

$542 \quad$ 10.1016/j.clim.2009.01.009.

543 Harris R, Olson AJ, Goodsell DS. 2008. Automated prediction of ligand-binding sites in

544 proteins. Proteins: Structure, Function and Genetics 70:1506-1517. DOI:

$545 \quad 10.1002 /$ prot.21645.

546 Hijiya N, van der Sluis IM. 2015. Asparaginase-associated toxicity in children with acute

547 lymphoblastic leukemia. DOI: 10.3109/10428194.2015.1101098. 
548 Hijiya N, van der Sluis IM. 2016. Asparaginase-associated toxicity in children with acute

549 lymphoblastic leukemia. Leukemia \& lymphoma 57:748-57. DOI:

550 10.3109/10428194.2015.1101098.

551 Huang Y, Niu B, Gao Y, Fu L, Li W. 2010. CD-HIT Suite: A web server for clustering and

552 comparing biological sequences. Bioinformatics 26:680-682. DOI:

553 10.1093/bioinformatics/btq003.

554

555

556

Huerta-Saquero A, Evangelista-Martínez Z, Angélica ME, Perez-Rueda E. 2013. Rhizobium etli asparaginase II: an alternative for acute lymphoblastic leukemia (ALL) treatment.

557

Isupov MN, Obmolova G, Butterworth S, Badet-Denisot M-A, Badet B, Polikarpov I, Littlechild

558

559

560

561

562

563

564

565

566

567

568

569

570

JA, Teplyakov A. 1996. Substrate binding is required for assembly of the active conformation of the catalytic site in Ntn amidotransferases: evidence from the 1.8 å crystal structure of the glutaminase domain of glucosamine 6-phosphate synthase. Structure 4:801810. DOI: 10.1016/S0969-2126(96)00087-1.

Kamal N, Koh C, Samala N, Fontana RJ, Stolz A, Durazo F, Hayashi PH, Phillips E, Wang T, Hoofnagle JH, Drug-Induced Liver Injury Network. 2019. Asparaginase-induced hepatotoxicity: rapid development of cholestasis and hepatic steatosis. Hepatology international 13:641-648. DOI: 10.1007/s12072-019-09971-2.

Karplus M, Kuriyan J. 2005. Molecular dynamics and protein function. Proceedings of the National Academy of Sciences of the United States of America 102:6679-85. DOI: 10.1073/pnas.0408930102.

Katz AJ, Chia VM, Schoonen WM, Kelsh MA. 2015. Acute lymphoblastic leukemia: an assessment of international incidence, survival, and disease burden. Cancer Causes \& 
572 Krishnapura PR, Belur PD, Subramanya S. 2016. A critical review on properties and applications 573 of microbial 1-asparaginases. Critical reviews in microbiology 42:720-37. DOI:

574

575

576

577

578

579

580

581

582

583

584

585

586

587

588

589

590

591

592

593 10.3109/1040841X.2015.1022505.

Lanvers-Kaminsky C. 2017. Asparaginase pharmacology: challenges still to be faced. Cancer Chemotherapy and Pharmacology 0:1-12. DOI: 10.1007/s00280-016-3236-y.

Lopes AM, Oliveira-Nascimento L de, Ribeiro A, Tairum CA, Breyer CA, Oliveira MA de, Monteiro G, Souza-Motta CM de, Magalhães P de O, Avendaño JGF, Cavaco-Paulo AM, Mazzola PG, Rangel-Yagui C de O, Sette LD, Converti A, Pessoa A. 2015. Therapeutic Lasparaginase: upstream, downstream and beyond. Critical Reviews in Biotechnology 8551:1-18. DOI: 10.3109/07388551.2015.1120705.

Magnan CN, Zeller M, Kayala MA, Vigil A, Randall A, Felgner PL, Baldi P. 2010. Highthroughput prediction of protein antigenicity using protein microarray data. Bioinformatics 26:2936-2943. DOI: 10.1093/bioinformatics/btq551.

Moreno-Enriquez A, Evangelista-Martinez Z, Gonzalez-Mondragon EG, Calderon-Flores A, Arreguin R, Perez-Rueda E, Huerta-Saquero A. 2012. Biochemical characterization of recombinant L-asparaginase (AnsA) from Rhizobium etli, a member of an increasing rhizobial-type family of L-asparaginases. Journal of microbiology and biotechnology $22: 292-300$.

Morris GM, Huey R, Lindstrom W, Sanner MF, Belew RK, Goodsell DS, Olson AJ. 2009. AutoDock4 and AutoDockTools4: Automated docking with selective receptor flexibility. Journal of Computational Chemistry 30:2785-2791. DOI: 10.1002/jcc.21256.

Nguyen HA, Su Y, Lavie A. 2016. Design and Characterization of Erwinia Chrysanthemi 1- 
594

595

596

597

598

599

600

601

602

603

604

605

606

607

608

609

610

611

612

613

614

615

616

Asparaginase Variants with Diminished 1-Glutaminase Activity. The Journal of biological chemistry 291:17664-76. DOI: 10.1074/jbc.M116.728485.

Nguyen HA, Su Y, Zhang JY, Antanasijevic A, Caffrey M, Schalk AM, Liu L, Rondelli D, Oh A, Mahmud DL, Bosland MC, Kajdacsy-Balla A, Peirs S, Lammens T, Mondelaers V, De Moerloose B, Goossens S, Schlicht MJ, Kabirov KK, Lyubimov A V, Merrill BJ, Saunthararajah Y, Van Vlierberghe P, Lavie A. 2018. A Novel 1-Asparaginase with low 1Glutaminase Coactivity Is Highly Efficacious against Both T- and B-cell Acute Lymphoblastic Leukemias In Vivo. Cancer research 78:1549-1560. DOI: 10.1158/00085472.CAN-17-2106.

Onciu M. 2009. Acute Lymphoblastic Leukemia. Hematology/Oncology Clinics of North America 23:655-674. DOI: 10.1016/j.hoc.2009.04.009.

Ortuño-Olea L, Durán-Vargas S. 2000. The L-asparagine operon of Rhizobium etli contains a gene encoding an atypical asparaginase. FEMS Microbiology Letters 189:177-182. DOI: 10.1016/S0378-1097(00)00275-5.

Pieters R, Hunger SP, Boos J, Rizzari C, Silverman L, Baruchel A, Goekbuget N, Schrappe M, Pui CH. 2011. L-asparaginase treatment in acute lymphoblastic leukemia. Cancer 117:238249. DOI: $10.1002 /$ cncr.25489.

Pui C-H, Relling M V., Downing JR. 2004. Acute Lymphoblastic Leukemia. New England Journal of Medicine 350:1535-1548. DOI: 10.1056/NEJMra023001.

Ramya LN, Doble M, Rekha VPB, Pulicherla KK. 2012. L-asparaginase as potent anti-leukemic agent and its significance of having reduced glutaminase side activity for better treatment of acute lymphoblastic leukaemia. Applied Biochemistry and Biotechnology 167:2144-2159. DOI: $10.1007 / \mathrm{s} 12010-012-9755-\mathrm{z}$. 
617 Roy A, Kucukural A, Zhang Y. 2010. I-TASSER: a unified platform for automated protein 618 structure and function prediction. Nature Protocols 5:725-738. DOI: 10.1038/nprot.2010.5.

619 Salzer W, Bostrom B, Messinger Y, Perissinotti AJ, Marini B. 2017. Asparaginase activity levels 620 and monitoring in patients with acute lymphoblastic leukemia. Leukemia and Lymphoma 621 0:1-10. DOI: 10.1080/10428194.2017.1386305.

622 Sanches M, Kraunchenko S, Polikarpov I. 2016. Structure, Substrate Complexation and 623

624 Reaction Mechanism of Bacterial Asparaginases. Current chemical biology 1:75-86. DOI:

625

626

627

628

629

630 $10.2174 / 187231307779814057$.

Sanner MF. 1999. Python: a programming language for software integration and development. Journal of molecular graphics \& modelling 17:57-61.

Santos AR, Pereira VB, Barbosa E, Baumbach J, Pauling J, Röttger R, Turk MZ, Silva A, Miyoshi A, Azevedo V. 2013. Mature Epitope Density - A strategy for target selection based on immunoinformatics and exported prokaryotic proteins. BMC Genomics 14:S4. DOI: 10.1186/1471-2164-14-S6-S4.

631

Schein PS, Rakieten N, Gordon BM, Davis RD, Rall DP. 1969. The toxicity of Escherichia coli 633 L-asparaginase. Cancer research 29:426-34.

Schwab C, Harrison CJ. 2018. Advances in B-cell Precursor Acute Lymphoblastic Leukemia 634 Genomics. HemaSphere 2:1. DOI: 10.1097/HS9.0000000000000053.

635 Sievers F, Wilm A, Dineen D, Gibson TJ, Karplus K, Li W, Lopez R, McWilliam H, Remmert 636 M, Söding J, Thompson JD, Higgins DG. 2011. Fast, scalable generation of high-quality 637 protein multiple sequence alignments using Clustal Omega. Molecular systems biology $638 \quad 7: 539$. DOI: $10.1038 / \mathrm{msb} .2011 .75$.

639 Singh SK, Cousens LP, Alvarez D, Mahajan PB. 2012. Determinants of immunogenic response 
640

641

642

643

644

645

646

647

648

649

650

651

652

653

654

655

656

657

658

659

660

661

662

to protein therapeutics. Biologicals 40:364-368. DOI: 10.1016/j.biologicals.2012.06.001.

Sturniolo T, Bono E, Ding J, Raddrizzani L, Tuereci O, Sahin U, Braxenthaler M, Gallazzi F, Protti MP, Sinigaglia F, Hammer J. 1999. Generation of tissue-specific and promiscuous HLA ligand databases using DNA microarrays and virtual HLA class II matrices. Nature Biotechnology 17:555-561.

Swain AL, Jaskólski M, Housset D, Rao JK, Wlodawer A. 1993. Crystal structure of Escherichia coli L-asparaginase, an enzyme used in cancer therapy. Proceedings of the National Academy of Sciences of the United States of America 90:1474-8. DOI:

10.1073/pnas.90.4.1474.

Thomas X, Le Jeune C. 2016. Erythrocyte encapsulated 1 -asparaginase (GRASPA) in acute leukemia. International Journal of Hematologic Oncology 5:11-25. DOI: 10.2217/ijh-20160002.

Walenciak J, Wyka K, Janczar S, Młynarski W, Zalewska-Szewczyk B. 2019. Dynamic changes in specific anti-L-asparaginase antibodies generation during acute lymphoblastic leukemia treatment. Pharmacological Reports 71:311-318. DOI: 10.1016/j.pharep.2018.11.002.

Wang P, Sidney J, Kim Y, Sette A, Lund O, Nielsen M, Peters B. 2010. Peptide binding predictions for HLA DR, DP and DQ molecules. BMC Bioinformatics 11:568. DOI: $10.1186 / 1471-2105-11-568$.

Wishart DS, Feunang YD, Guo AC, Lo EJ, Marcu A, Grant JR, Sajed T, Johnson D, Li C, Sayeeda Z, Assempour N, Iynkkaran I, Liu Y, Maciejewski A, Gale N, Wilson A, Chin L, Cummings R, Le D, Pon A, Knox C, Wilson M. 2018. DrugBank 5.0: a major update to the DrugBank database for 2018. Nucleic Acids Research 46:D1074-D1082. DOI: $10.1093 / \mathrm{nar} / \mathrm{gkx} 1037$. 
$663 \mathrm{Xu} \mathrm{D}$, Zhang Y. 2011. Improving the physical realism and structural accuracy of protein models 664 by a two-step atomic-level energy minimization. Biophysical journal 101:2525-34. DOI: $665 \quad$ 10.1016/j.bpj.2011.10.024.

666 Zhang Y. 2008. I-TASSER server for protein 3D structure prediction. BMC bioinformatics 9:40. 667 DOI: $10.1186 / 1471-2105-9-40$.

668 


\section{Table $\mathbf{1}$ (on next page)}

Table 1. Representative Streptomyces ASNases of the PF00710.11 and PF06089.11 families, at $60 \%$ identity cutoff. 
1 Table 1. Representative Streptomyces ASNases of the PF00710.11 and PF06089.11

2 families, at $60 \%$ identity cutoff.

\begin{tabular}{|l|l|l|l|}
\hline ASNase ID & Organism & $\begin{array}{l}\text { Length } \\
\text { (amino } \\
\text { acids) }\end{array}$ & Family \\
\hline PF00710.11 family & \multicolumn{2}{l|}{} \\
\hline WP_053609500.1 & S.purpurogeneiscleroticus & 373 & PF00710.11 \\
\hline WP_053610569.1 & S. purpurogeneiscleroticus & 338 & PF00710.11 \\
\hline WP_055617501.1 & S. phaeochromogenes & 380 & PF00710.11 \\
\hline WP_051815467.1 & S. lavenduligriseus & 363 & PF00710.11 \\
\hline WP_078649241.1 & S. fradiae & 350 & PF00710.11 \\
\hline EFL23513.1 & S. himastatinicu ATCC 53653 & 351 & PF00710.11 \\
\hline WP_014151616.1 & S. cattleya & 331 & PF00710.11 \\
\hline WP_095730579.1 & S. albidoflavus & 333 & PF00710.11 \\
\hline WP_078965752.1 & S. aureocirculatus & 343 & PF00710.11 \\
\hline WP_078513220.1 & S. purpureus & 421 & PF00710.11 \\
\hline WP_009718687.1 & S. himastatinicus & 347 & PF00710.11 \\
\hline WP_079189481.1 & S. paucisporeus & 384 & PF00710.11 \\
\hline WP_052425051.1 & S. fulvoviolaceus & 340 & PF00710.11 \\
\hline ELP65653.1 & S. turgidiscabies Car8 & 358 & PF00710.11 \\
\hline WP_070201703.1 & S. nanshensis & 347 & PF00710.11 \\
\hline KWW98572.1 & S. thermoautotrophicus & 333 & PF00710.11 \\
\hline WP_073950513.1 & S. kebangsaanensis & 333 & PF00710.11 \\
\hline WP_030748190.1 & S. griseus & 329 & PF00710.11 \\
\hline WP_059134811.1 & S. alboniger & 332 & PF00710.11 \\
\hline PF06089.11 family & & & \\
\hline ARZ68596.1 & S. albireticuli & 428 & PF06089.11 \\
\hline CDR15801.1 & S. iranensis & 387 & PF06089.11 \\
\hline SOD64826.1 & S. zhaozhouensis & 316 & PF06089.11 \\
\hline WP_020554088 & S. scabrisporus & 332 & PF06089.11 \\
\hline WP_044373749 & S. ahygroscopicus & 330 & PF06089.11 \\
\hline WP_078645645 & S. varsoviensis & 348 & PF06089.11 \\
\hline WP_078980718.1 & S. scabrisporus & PF06089.11 \\
\hline
\end{tabular}




\section{Table 2 (on next page)}

Table 2. High-affinity epitope prediction. Epitope number, CPR value, allele coverage, and ED of ASNases. 
1 Table 2. High-affinity epitope prediction. Epitope number, CPR value, allele coverage, 2 and ED of ASNases.

\begin{tabular}{|c|c|c|c|c|}
\hline ASNase ID & Epitope number & CPR value & Allele number & ED \\
\hline P00805_EcAll & 10 & 0.6383 & 5 & 0.0114 \\
\hline WP_053609500.1 & 12 & 0.5174 & 5 & 0.0171 \\
\hline WP_053610569.1 & 14 & 0.5381 & 8 & 0.0196 \\
\hline WP_055617501.1 & 7 & 0.4532 & 7 & 0.0112 \\
\hline WP_051815467.1 & 6 & 0.6673 & 5 & 0.0060 \\
\hline WP_078649241.1 & 7 & 0.4554 & 6 & 0.0111 \\
\hline EFL23513.1 & 10 & 0.6054 & 8 & 0.0115 \\
\hline WP_014151616.1 & 3 & 0.4024 & 5 & 0.0056 \\
\hline WP_095730579.1 & 8 & 0.5346 & 2 & 0.0115 \\
\hline WP_078965752.1 & 3 & 0.4987 & 4 & 0.0045 \\
\hline WP_078513220.1 & 9 & 0.4551 & 6 & 0.0119 \\
\hline WP_009718687.1 & 5 & 0.6480 & 4 & 0.0052 \\
\hline WP_079189481.1 & 4 & 0.5217 & 5 & 0.0051 \\
\hline WP_052425051.1 & 10 & 0.4369 & 6 & 0.0170 \\
\hline ELP65653.1 & 5 & 0.6717 & 3 & 0.0047 \\
\hline WP_070201703.1 & 7 & 0.6637 & 6 & 0.0069 \\
\hline KWW98572.1 & 4 & 0.7254 & 4 & 0.0034 \\
\hline WP_073950513.1 & 6 & 0.7424 & 4 & 0.0048 \\
\hline WP_030748190.1 & 3 & 0.5125 & 6 & 0.0046 \\
\hline Q9K4F5_ScAll & 3 & 0.4167 & 3 & 0.0053 \\
\hline ARZ68596.1 & 5 & 0.6283 & 5 & 0.0044 \\
\hline SOD64826.1 & 5 & 0.5046 & 5 & 0.0080 \\
\hline WP_078645645.1 & 7 & 0.6404 & 5 & 0.0074 \\
\hline CDR15801.1 & 5 & 0.5510 & 5 & 0.0059 \\
\hline WP_078980718.1 & 6 & 0.7003 & 6 & 0.0056 \\
\hline WP_044373749.1 & 3 & 0.7114 & 4 & 0.0027 \\
\hline
\end{tabular}


3 Epitope number refers to the number of epitopes with CPR $<1$. Allele number is the 4 number of allele coverage for high affinity epitopes (with a CPR $<1$ ). 5 


\section{Table 3(on next page)}

Table 3. SsAll-2 putative binding site residues. PF06089.11 family conserved residues are shown in bold. 
1 Table 3. SsAll-2 putative binding site residues. PF06089.11 family conserved residues

2 are shown in bold.

\begin{tabular}{|c|c|c|}
\hline Site & $\begin{array}{l}\text { Ligand-binding positions predicted by } \\
\text { AutoLigand }\end{array}$ & $\begin{array}{l}\text { Ligand-binding positions } \\
\text { predicted by blind molecular } \\
\text { docking }\end{array}$ \\
\hline
\end{tabular}

Arg 58, Ser 59, Lys 62, Asn 141, Ser 143, A Gly 144, Lys 145, His 146, Ala 147, Gly 236, Gly 237, Asp 238, Gly 239, Lys 255, Gly 256, Gly 257, Ala 258, Pro 281, Leu 326

Asn 135, Thr 136, Arg 137, Arg 139 , Asn 141, Gly 144, His 146, Asp 192

Ala 85 , Gly 86 , Ser 87 , His 88 , Thr 89 , Gly

B 90, Gln 91, His 94, Leu 164, Asp 165, Pro 166, Gly 167, His 168, Leu 173, Glu 177, Gly 178, Asp 180 


\section{Table 4(on next page)}

Table 4. Molecular docking energies of ASNases.

Table 4. Molecular docking energies of ASNases. 
1 Table 4. Molecular docking energies of ASNases.

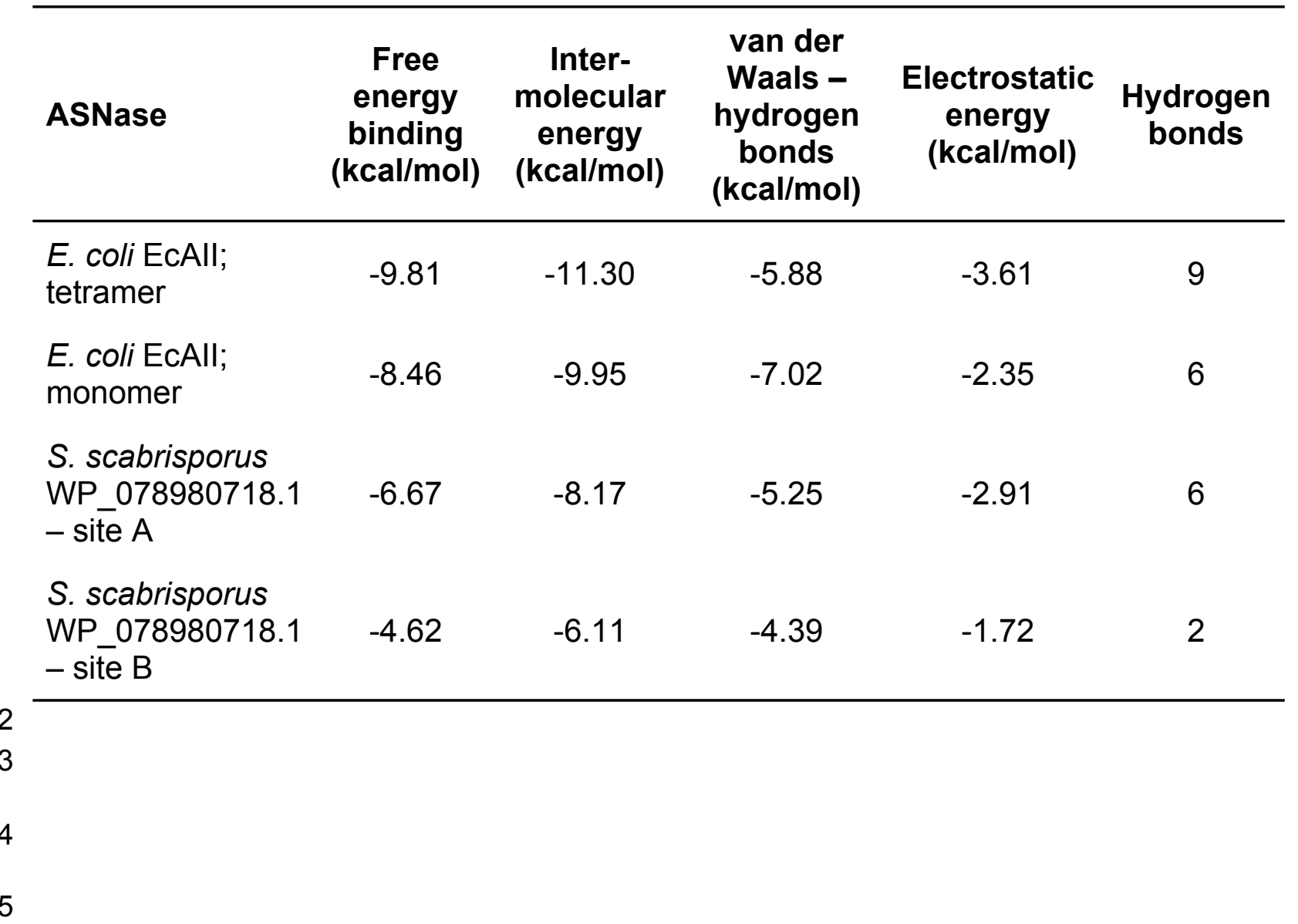


Figure 1

Phylogenetic tree of PF00710.11 (A) and PF06089.11 (B) families.

Blue dots highlight reference sequences added to each analysis. Red dots highlight sequences used as internal controls (asparaginases from E. coli and R. etli, respectively). A total of 1000 replicates were performed. Bootstrap values are indicated.

A

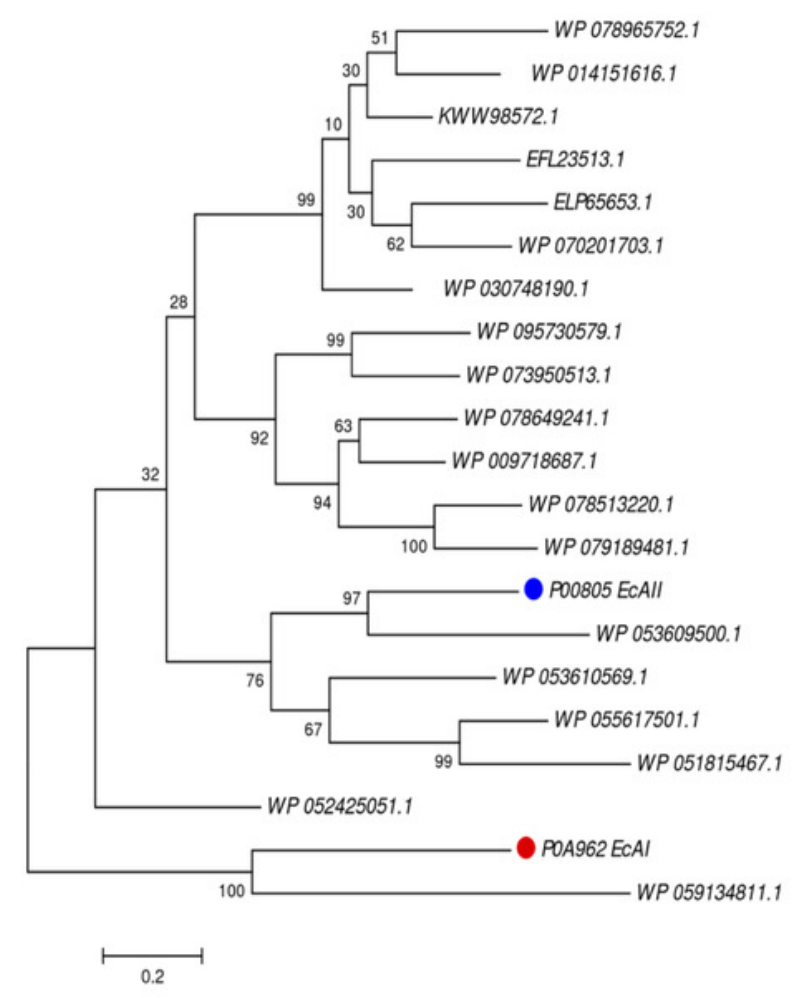

B

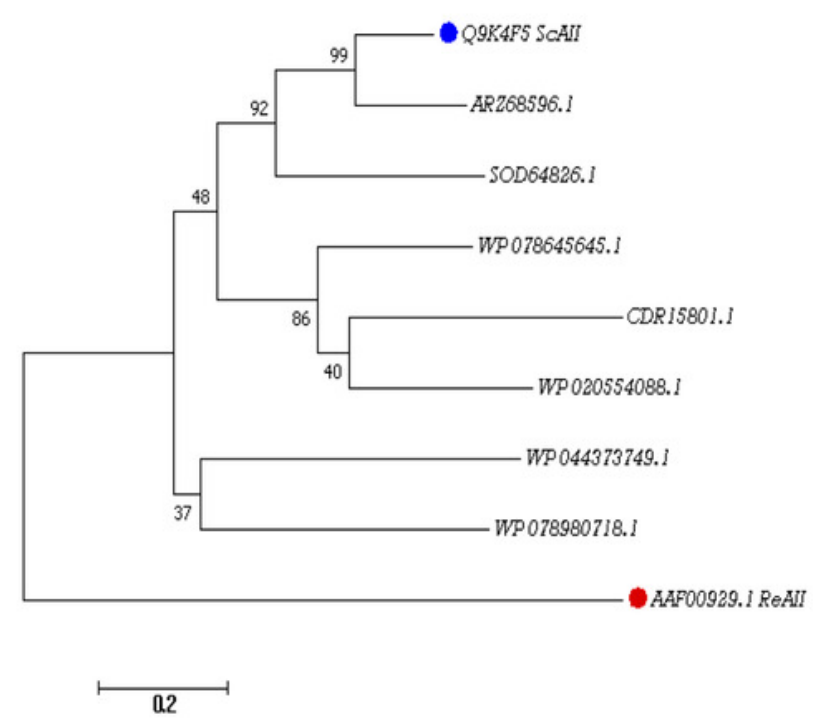


Figure 2

ASNase antigenicity predictions.

The antigenicity scores for PF00710.11 family (A) and PF06089.11 family (B) of Streptomyces asparaginases were compared with the EcAll antigenicity score. 

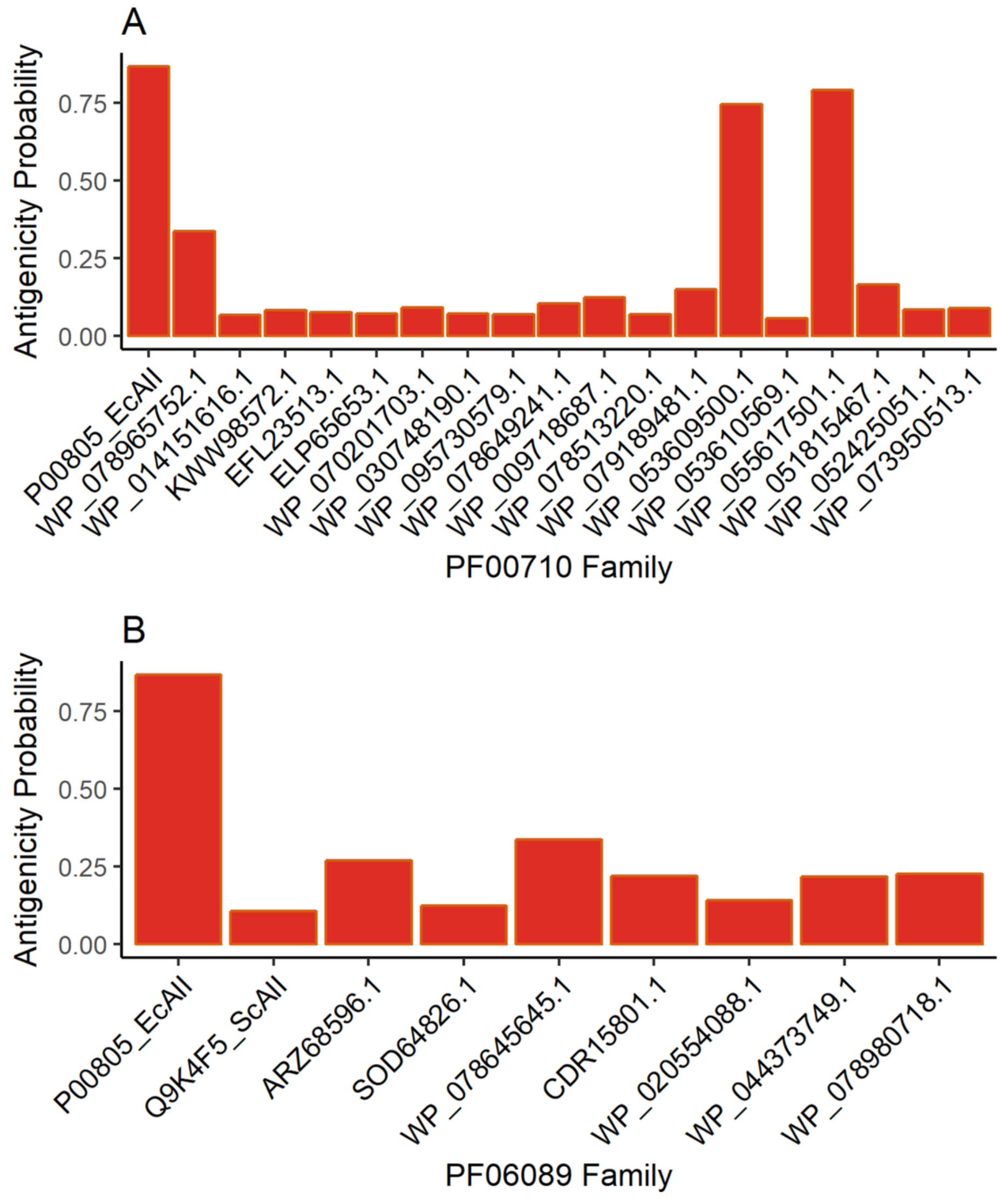
Figure 3

Epitope mapping of ASNases of the PF familes evaluated, (A) PF00710.11 and (B) PF06089.11.

The epitopes identified along with the ASNase sequences are shown. The color intensity represents the number of hits for each of them.

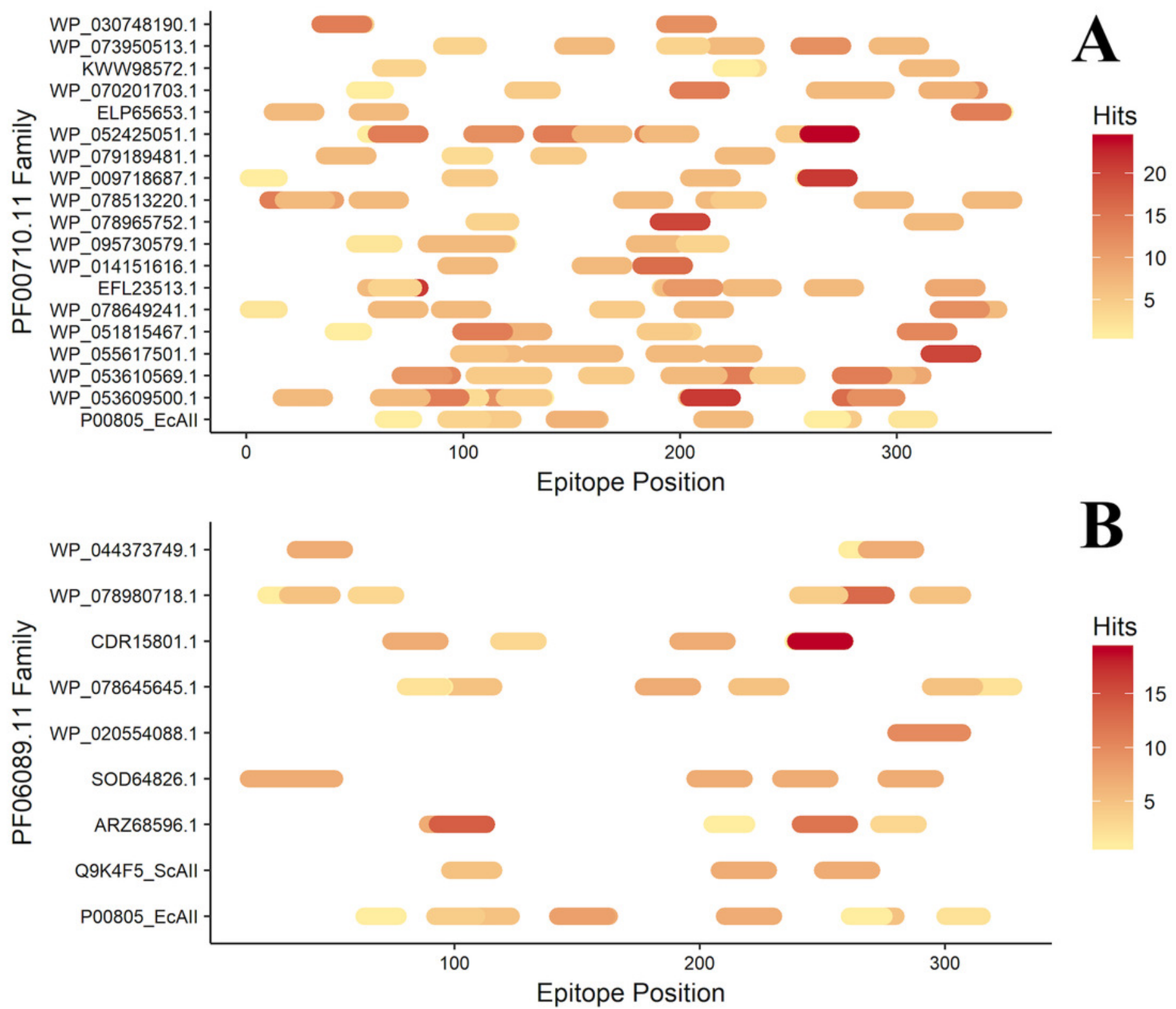


Figure 4

3D protein structure prediction of S. scabrisporus asparaginase II (WP_078980718.1; SsAll-2).

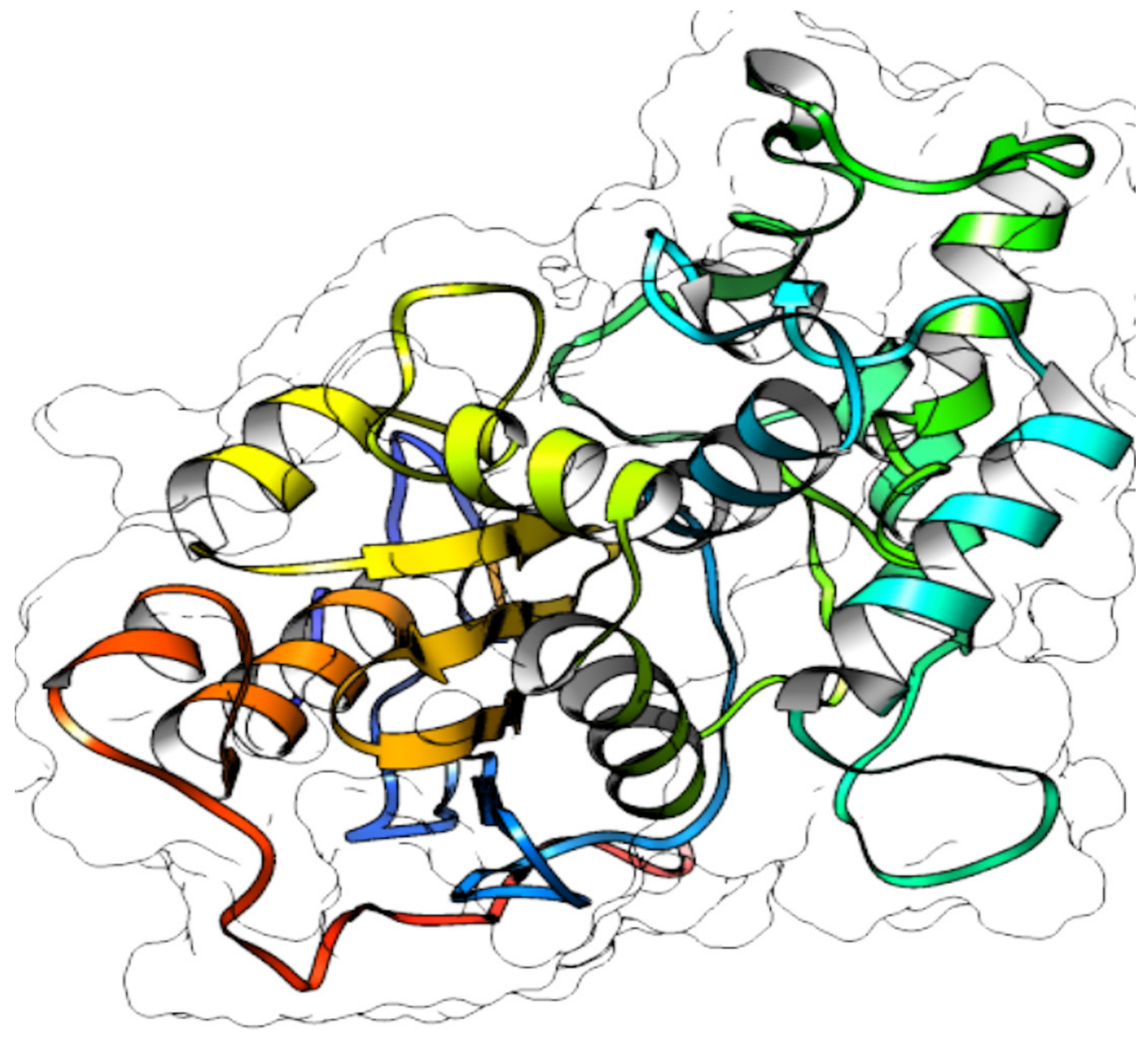


Figure 5

SsAll-2 putative binding sites.

A) Site A (orange) contains the NCSGKHXAML sequence and site B (blue) contains the SHTGQxHFV motif. B) Residues involved with asparagine through a direct interaction, obtained by blind molecular docking.

A

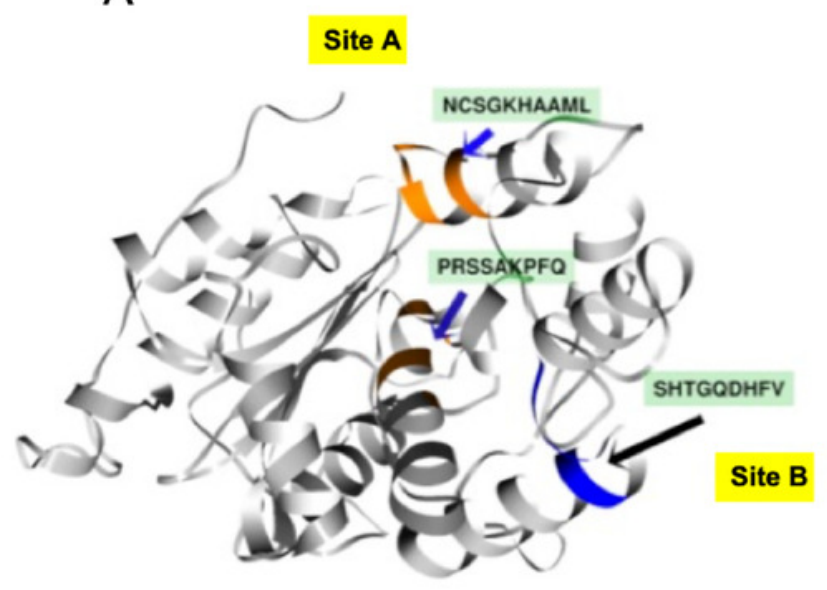

B

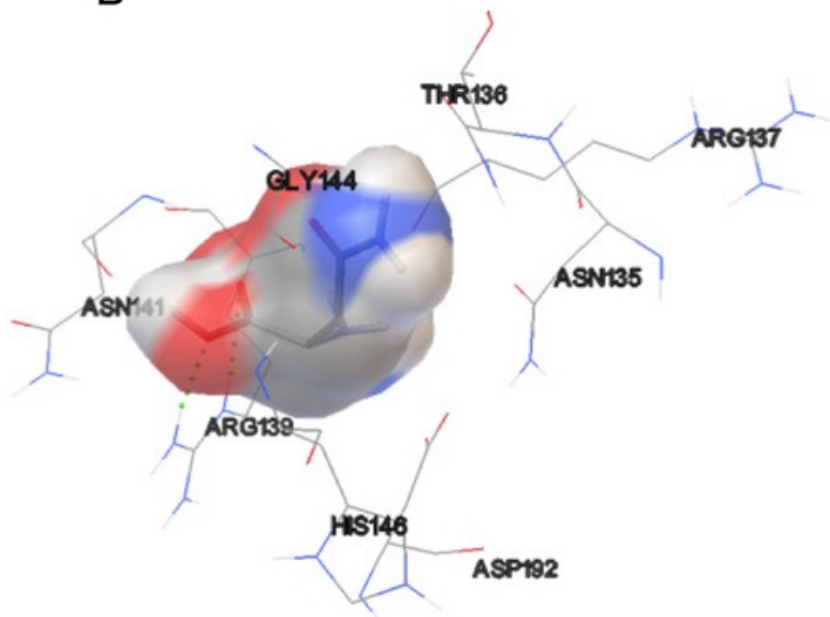




\section{Figure 6}

EcAll dimer AutoLigand analysis.

EcAll subunit $A$ is shown in cyan and subunit $C$ in magenta. The red mesh represents the highest-affinity pocket found by AutoLigand (putative active site). The site represented in the scheme corresponds to the residues located at a maximum distance of $5 \AA$ using 20 points: Thr 12, Tyr 25, Ser 58, Gln 59, Thr 89, and Asp 90 from subunit C and Asn 248 and Glu 283 from subunit $A$.

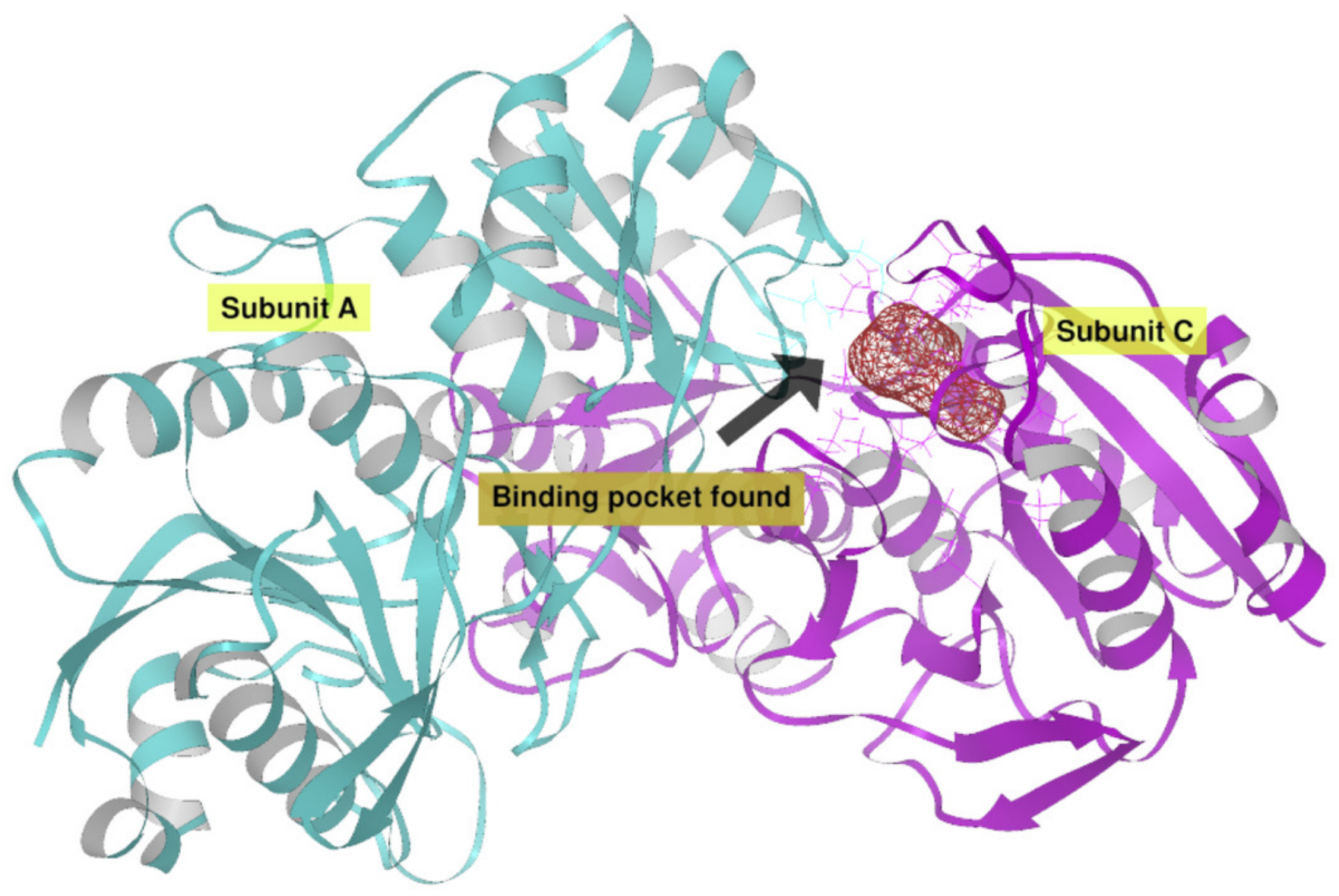




\section{Figure 7}

AutoLigand results for EcAll.

The minima observed in the total energy graphs per unit volume represent putative binding sites in the structures analyzed, for the monomer, dimer, and tetramer conformation. As more filling points are used, the binding sites, cavities, or grooves are filled and the affinity decreases. The best sites are the ones with the lowest energy and the lowest volume. 

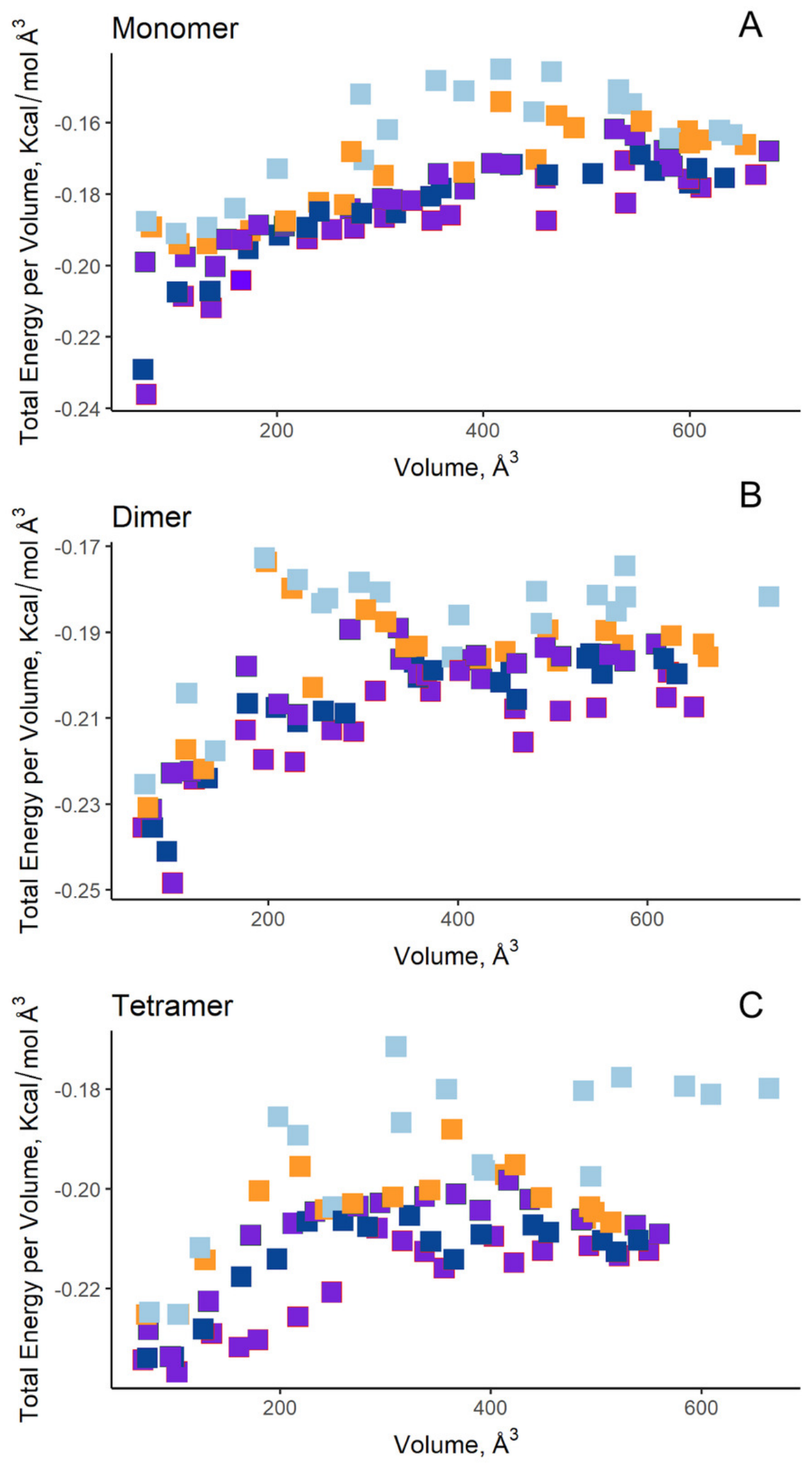
Figure 8

Interaction maps for sites A and B from S. scabrisporus ASNase.

The black spheres represent carbon atoms, the blue nitrogen and the red oxygen. Hydrogen bonds are represented by green dotted lines and hydrophobic interactions are shown as red half-moons.

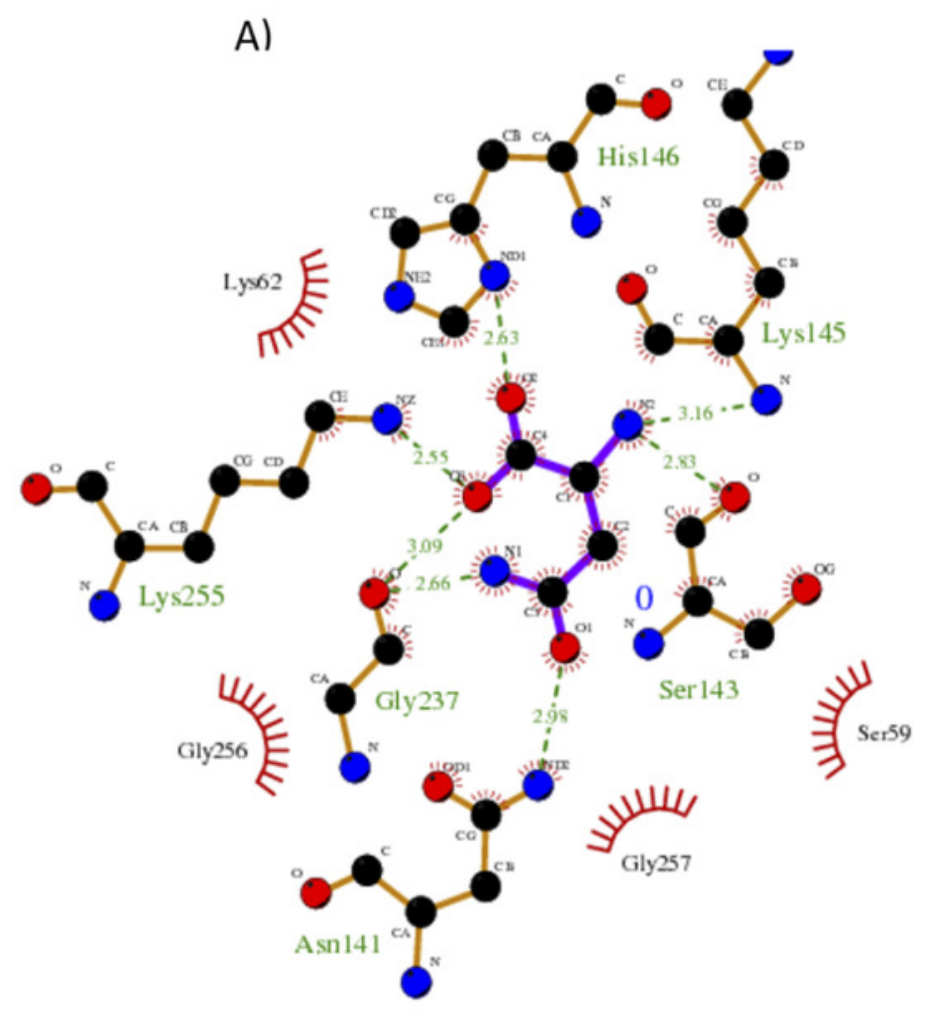

B)

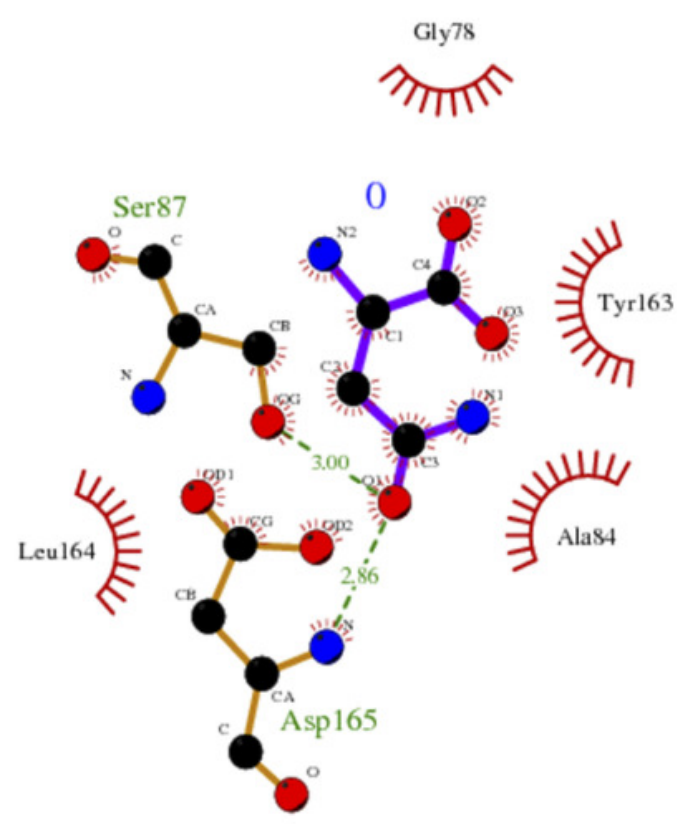

